Alkeste 21. Juli 9m.8-9m.9, Bilder verwaschen; 22. Juli 9m.8, Luft gut; 23. Juli 9m.8, Wolken; 24. Juli 9m.6, zuletzt aber wegen Wolken sehr schwach;26. Juli theilweise durch Wolken beobachtet, Aug. 4. sehr nahe am Mond, gegen den Vergl. 9.0 erschion sie $10^{\mathrm{m}} .0-10^{\mathrm{m}} .5$, Beobachtung etwas unsicher; 11 . Aug. $10^{\mathrm{m}} .0$, Bilder wegen starken Dunst äusserst unruhig; 12. Aug. bei Mondschein $9^{\mathrm{m}} .8-10^{\mathrm{m}} .0$, Luft gut; 13. Aug. 10 10 , Bilder unruhig; 14. Augr. durch starke Musik in einem Nachbargarten fortwährend gestört.

Fides 28. Juli 10m.5, Luft schlecht, Gewitter, Vergl. * selır unruhig; 6. Aug. in Mondnähe 10m.5, Cirri. unrulige Bilder; 7. Aug. 10 $\mathrm{m} .5$, zuletzt aber sehr schwach, Wolkenschleier; 8 . Aug. $10^{\mathrm{m} .5}$, zuletzt wegen

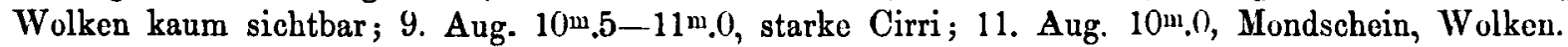

Flora 6. Aug. $8^{\mathrm{m}} .5-8^{\mathrm{n}} .8$, sehr verwaschen, nahe am Vollmond.

Pandora 7. Aug. $10^{\mathrm{m}} .0-10^{\mathrm{m}} .5$, nahe am Mond, zuletzt Wolken; 12. Aug. 9m.8, Luft gut.

Lureley 12. Aug. 10m.5, Mond, Luft ausgezeiclinet; 13. Aug. Luft gut; 14. Aug. $10^{\mathrm{m}} .5$ Mond; 15. Aug. 10m.5$11^{\mathrm{m}} ; 16$. Aug. $10^{\mathrm{m}}-11^{\mathrm{m}}$, Fadenbeleuchtung schlecht; 18. Aug. $10^{\mathrm{m}} .5$, Luft gut, Beleuchtung schlecht ; 25. Aug. lim, Luft schlecht, durch Wolken beobachtet; 4. Sept. sehr schwach, Mond, Wolken; 6. Sept. $10^{\mathrm{m}} .5$, dünner Wolkenschleier; 11 . Sept. $11^{\mathrm{m}}$, Wolken, Luft schlecht; 7. Oct. $12^{\mathrm{m}} .5$, Bilder mittelmässige, einzelne Wolken, 12. Oct. Bilder selır schlecht und schwach, dicker Nebel; 14 . Oct. $12^{\mathrm{m}} .5-13^{\mathrm{m}}$, zuletzt im Nebel verschwunden; 22. Oct. $12^{\mathrm{m}} \cdot-13^{\mathrm{n}}$, undeutliche Bilder, starker Sturm.

Lomia 15. Aug. $11^{\mathrm{m}}$, Decl.-Beobachtung unsicher; 16. Aug. $11^{\mathrm{m}}$.

Lydia 6. Sept. $10^{\mathrm{m}}$.() durch Wolken.

Galatea 1. Oct. $3^{\mathrm{m}} .5$ Mond nahe, Bilder schlecht; 17. Oct. 9m.6.

Clio 5. Oct, $9^{\mathrm{m}} .6-9^{\mathrm{m}} .8$, Mond, Cirri ; 6. Oct. $9^{\mathrm{m}} .8$, Bilder leidlich.

Amphitrite 5. Oct. $8^{\mathrm{m}} .6$ durch dichtes Gewölk, zuletzt ganz trübc.

Zelia 7. Oct. $11^{\mathrm{m}} .0$, sehr nebelig; 10. Oct. $11^{\mathrm{m}} .0$ öfters Wolken; 12. Oct. durch Wolken $11 ;{ }^{\text {m1 }} 14$. Oct. etwas heller als $11^{\mathrm{m}} ; 16$. Oct. $11^{\mathrm{m}}$, wegen Nebel schwer sichtbar; 17. Oct. $11^{\mathrm{m}}$, Bilder gut; 22. Oct. 10m.5; 23. Oct. $10^{\mathrm{m}} .5$, Wolken, zuletzt ganz trübe; 5 . Decbr. $11^{\mathrm{m}} .5$ durch Dunst.

Sibylla 14. Oct $11^{\mathrm{m}} .5$, Bilder mittelmässig; 16. Oct. $11^{\mathrm{m}},-12$, Bilder schlecht, Nobel; 17. Oct. $11^{\mathrm{m}}$, Isuft gut; 18. Oct. $11^{\mathrm{m}} .0-11^{\mathrm{m}} .5$, Luft sehr sehlecht, Sturm; 22. Oct. 11 ${ }^{\mathrm{m}}$, Sturm; 23. Oct. ganz verschleiert, obwohl $11^{\mathrm{m}} ; 16$. Novbr. $12^{\mathrm{m}}$, im Dunst verschwunden.

Cybele 18. Oct. $10^{\mathrm{m}} .0$, Sturm.

Phocaea 30. Oct. 9m.8, Vollmond, zuletzt dicke Wolken; 6. Nov. 9m.5, Bilder unruhig.

Mnemosyne 30. Oct. $10^{\mathrm{m}}$, nahe am Vollmond; 4. Nov. $10^{\mathrm{m}} .5$.

Sirona 4. Nov. $11^{\mathrm{m}} .2$, wegen Mond sehr schwach; 6. Nov. $11^{\mathrm{m}}, 0$.

Amalthea 14. Nov. $12^{\mathrm{m}} .5$, Nebel; 15. Nov. $12^{\mathrm{m}} .5$, durch Wolken beobachtet; 16. Nov. 12m.0, Luft veründerlich oft sehr gut.

Velleda 15. Nov. $11^{\mathrm{m} 5}$, Sturm; 16. Nov. 11m, öfter durch Wolken bcobachtet; 29. Nov. 11m.2, Mondschein, fast immor durch Wolken beobachtet.

Antigone 7. Decbr. $11^{\mathrm{m}}-11^{\mathrm{m}} .5$, wegen Wolken schwer sichtbar.

P. bedeutet als Beobachter Herr Peter, B. Bruhns,

Leipzig im März 1877.

Brulins.

\title{
Aus einem Schreiben des Herrn Prof. Strasser an den Herausgeber.
}

Die grosse Differenz der Beobachtung der Euterpe, von Wien und Kremsmünster (A. N. No. 2131) veranlasste eine Untersuchung des Grundes. Hierbei stellte sich heraus, dass die Uhr-Correction verkehrt angebracht wurde, und bei der Bestimmung der Declination in den Einheiten der Minuten statt 3 die Ziffer 5 gelesen wurde.
Vermöge dieser Bemerkungen stellt sich dic AR. um 6.42, und die Declination um 2 niederer. Wesshalb es heissen soll $a=9^{\mathrm{h}} 35^{\mathrm{m}} 27^{\mathrm{s}} .65$ und $g^{\mathrm{s}}=+16^{0} 55^{\circ}$ $21^{\prime \prime} .0$ um $10^{\mathrm{h}} 43^{\mathrm{m}} 28^{\mathrm{s}} .40$.

Kremsmünster den 24. Mai 1877.

(j. Strasser. 